\title{
Complex suicide of a drug addict; a case report
}

\author{
Sanjeewa $\mathrm{HKR}^{1}$, Vidanapathirana $\mathrm{M}^{2 *}$
}

\begin{abstract}
The term "complex suicide" refers to suicides in which more than one method is applied. It can be further classified as planned and unplanned complex suicide. In planned complex suicides, two or more methods are employed simultaneously in order to make sure that death will occur even if one method fails. In unplanned complex suicides, several other methods of suicide are tried after the first method chosen fails. Following case discussion is based on a death with two methods of suicide. Body of a 20 year old drug addict was recovered from a river. A blood stained table knife was found in his room. There were no features of struggle at scenes. He had no previous attempts of suicide. Autopsy examination revealed four (4) superficial and parallel cut throat injuries. There were no evidence of haemorrhagic shock, blood aspiration or air embolism. Fine continuous froth with kissing hyper inflated lungs were found. The cause of death was given as drowning. Since two suicidal methods were employed, namely cut throat and jumping in to river, it was identified as a case of complex suicide. Cut throat injuries could have been inflicted before entering the water. It appeared that, the initial cut throat injuries had failed to cause death and the victim subsequently succeeded by jumping in to river. Therefore, the death discussed showed clear characteristics of an unplanned complex suicide.
\end{abstract}

Key words: complex suicide, planned or unplanned, cut throat, drowning

\section{Full paper}

\section{Introduction}

Suicides are divided into simple and complex suicides. The term "complex suicide" refers to suicides in which more than one suicide method is applied. ${ }^{[1]}$ It is further classified as planned (primary) and unplanned (secondary) complex suicides.

In planned complex suicides, two or more methods are employed simultaneously in order to make sure that death will occur even if one method fails. ${ }^{[2]}$ Therefore, in planned complex suicides, typically two of the generally common methods of suicide are combined such as drowning, ingestion of medicinal

${ }^{1}$ Postgraduate trainee, ${ }^{2}$ Professor, Department of Forensic Medicine, Faculty of Medical Sciences, University of Sri Jayewardenepura, Sri Lanka

*Corresponding author: Vidanapathirana M, E mail: mudithavidana@sjp.ac.lk, Tel: 0094772988227, Department of Forensic Medicine, Faculty of Medical Sciences, University of Sri Jayewardenepura, Sri Lanka

DOI: http://doi.org/10.4038/mljsl.v5i1.7350

https://orcid.org/0000-0003-0071-0996 drugs, hanging, use of firearms and jumping from a height. $^{[2]}$

In unplanned complex suicides, several other methods of suicide are tried after the first method chosen fails. ${ }^{[3]}$ Injuries by sharp force, especially cutting the wrists, are often found as the first act of suicide. In some cases, the suicidal method changes from the infliction of cut wounds in the wrists to the infliction of stab wounds on the area of chest overlying the heart. Other frequently used methods after failure of the first method are hanging and jumping from a height. ${ }^{[3]}$ Following case discussion is on a death with two methods of suicide.

\section{Case report}

Body of a 20 year old unmarried male was recovered from a river. According to police, he was a drug addict. According to mother, he had cut his neck at home and then jumped in to the river. A blood stained table knife was found in his room. There were 
no features of struggle at scenes. The body was recovered 24 hours after the initial incident.

He had no previous history of suicidal attempts. Autopsy examination revealed that the deceased was an average built person. His dominant hand was right and there were several superficial, multiple, parallel scars on front aspect of the left forearm (Fig. 1). However, there were no macro or microscopic evidence such as venipuncture sites, lung fibrosis, foreign body granulomas etc. There were four (4) cut injuries on the front aspect of the neck (Fig. 2). They were superficial and parallel. They were not associated with deep injuries on structures such as strap muscles, airways or neck vessels.

Cutis anserina and washer woman skin/ sign were found. Fine continuous froth was found at nose and mouth. Lungs were hyper inflated (Fig. 3). There were no defense injuries. According to histopathological studies, there were foreign bodies beyond secondary bronchi (Fig. 4 photomicrograph) and the other organs did not show any remarkable findings. Toxicological analyses of blood and bile were negative for common poisons, alcohol, narcotics and drugs. The cause of death was given as drowning.

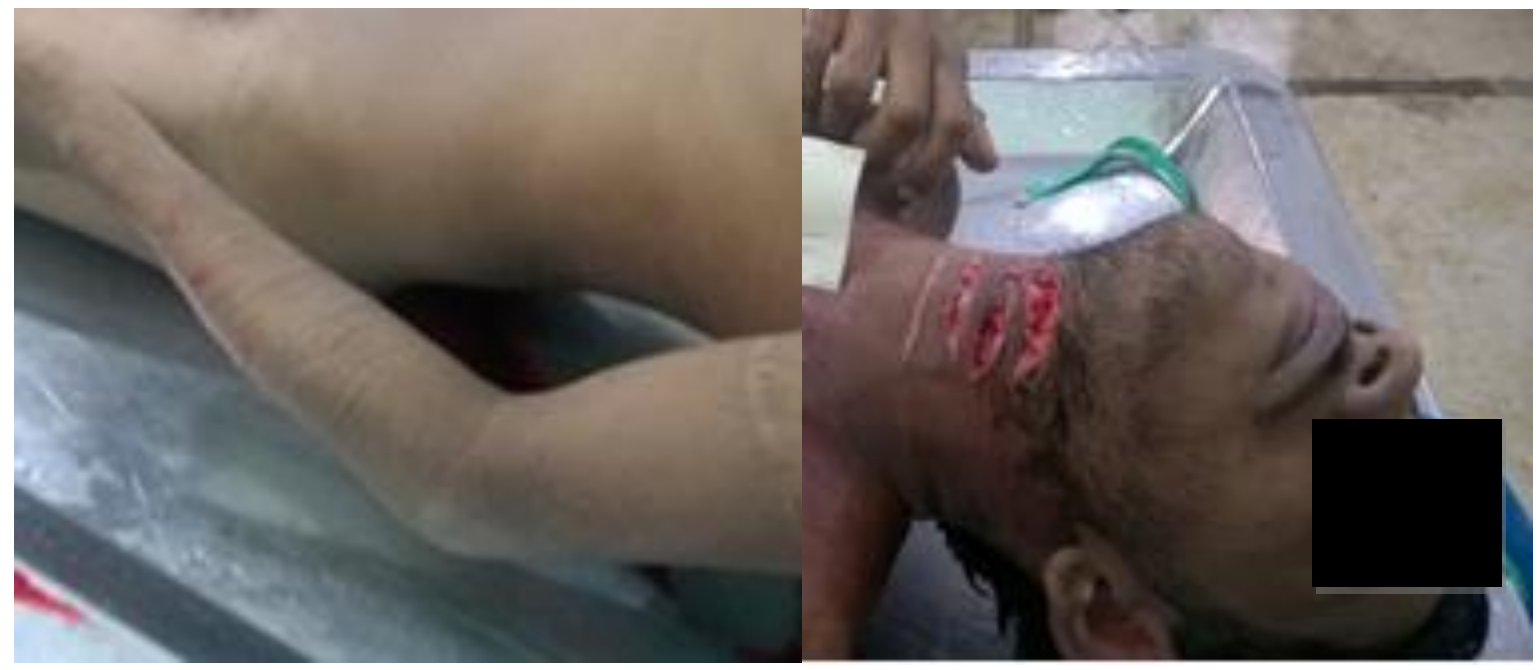

Figure 1: Multiple scars on his left forearm. Figure 2: Multiple, parallel and superficial cuts

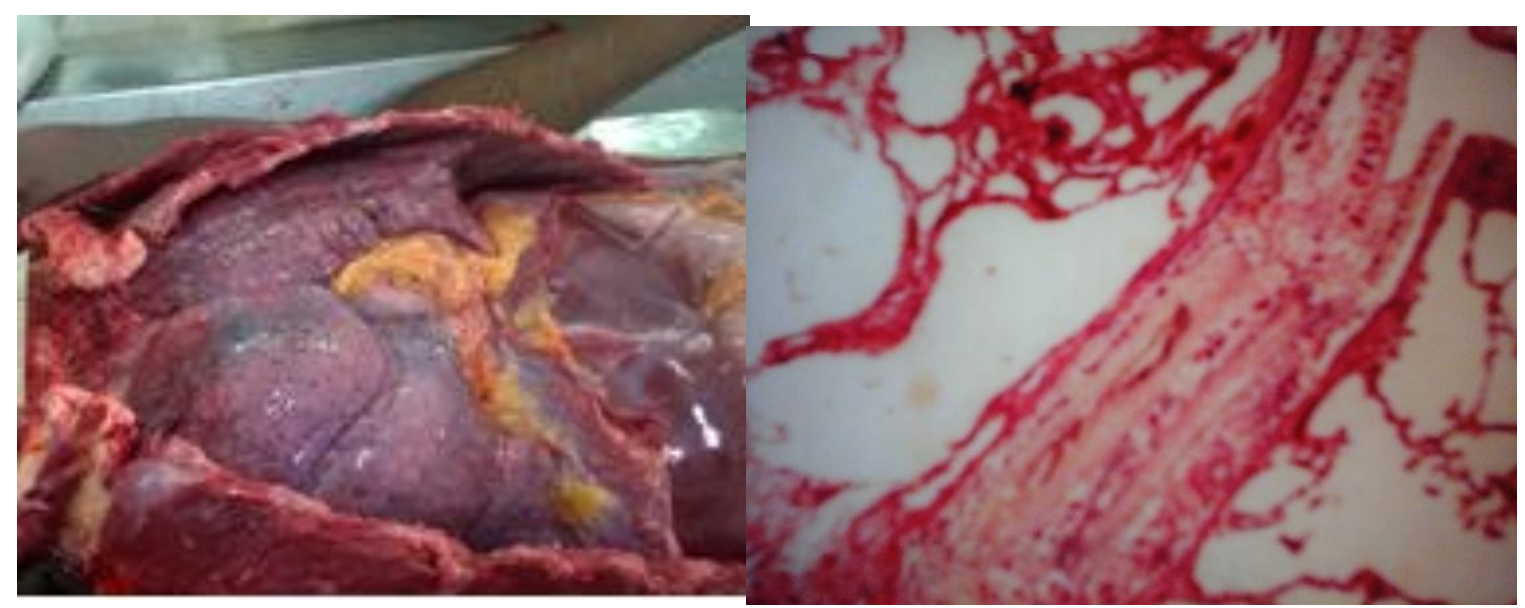

Figure 3. Hyper inflated lungs Figure 4: Foreign bodies (arrow) beyond secondary bronchi(H and $\mathrm{E}$ stain $\mathrm{x} 40$ ) 


\section{Discussion}

There were four superficial, parallel cut injuries on the front aspect of the neck. The injuries caused by propellers, animal predators or water monitors can mimic cut throat injuries in bodies recovered from water. However, in postmortem animal bites, in addition to teeth injuries, there are associated claw marks and they are haphazardly placed. Therefore, presence of teeth and claw marks help to identify postmortem animal bites ${ }^{[4]}$ and in this case, absence of teeth or claw marks excluded the possible postmortem animal bites. Though propellers could cause multiple, parallel cuts in the body, the presence of such injuries at an elective site of suicide favoured ante-mortem cut throat injuries and should have been received before entering to waterand it further confirmed the eye witness evidence.

Cut throat injuries are found in different circumstances; accidental, suicidal or homicidal. ${ }^{[5]}$ The distinction between homicidal, suicidal and accidental cut throat injuries is not an easy task for forensic pathologists who need to evaluate neck injuries case by case. Thus, scene investigation findings, the deceased's medical history as well as all autopsy and toxicological findings must be considered with skeptical approach before being able to establish the manner of death. ${ }^{[6]}$

Homicidal cut throats are usually associated with defense or bizarre type of injuries and such injuries were not found in this case. Whereas suicidal cut injuries are usually multiple, parallel and superficial and are found at elective sites such as neck as found in this case. ${ }^{[5]}$ Further, a blood stained knife was recovered from his room.

Though the suicidal knife injuries on the neck can be cuts or stabs, in this case, there were only cut injuries. ${ }^{[7]}$ The suicidal cut throats accompany with tentative or hesitant cuts or stabs and are multiple, almost parallel and superficial. ${ }^{[5]}$ Hesitation cuts are usually found in wrists, front of the neck and less commonly in the elbow or on the ankles. ${ }^{[8]}$ In this case, out of 4 cut throat injuries, the lowest, very superficial cut could be considered as a hesitant cut.
In homicidal cut throat injuries, the scene is usually disturbed and the weapon is usually taken away by the perpetrator whereas in suicidal cut throat injuries, the scene is undisturbed and the causative weapon is usually found at the scene as found in this case. ${ }^{[5]}$

Suicidal deaths are more commonly associated with psychiatric illness, substances abuse or history of previous suicidal attempts. In this case, though he did not have any previous suicidal attempts, he was a drug addict and there were scars of several previous self-inflicted injuries. Considering above features, the circumstance of his cut throat was ascertained as suicide.

However, the neck injuries did not penetrate deep structures and there were no major vessel or airway damage. Therefore, there were no autopsy evidence of mechanisms of death of cut throat injuries such as haemorrhagic shock, air embolism or aspiration of blood.

When a body is recovered from water, to diagnose drowning as the cause of death, we need to exclude fatal injuries or natural diseases that occur before or after entering the water and the toxicology screening also should be negative. There were no macroscopic or microscopic evidence of any significant natural pathology. The toxicology for alcohol, common poisons, narcotics and drugs were negative. Further, the diatom test was negative. However, the fine froth with inflated kissing lungs and microscopic evidence of foreign bodies beyond the secondary bronchi indicated that emphysema aquosum is a mechanism of death in this case. Foreign matter beyond secondary bronchi in a non-putrefied body may indicate that the person was alive when he entered water. Further, considering the history, eye witness, scene and autopsy findings, the manner of this immersion was ascertained as suicide.

When two potential modes of deaths are present, namely cut throat and jumping in to river, it is important to ascertain the real cause of death. In this case, there were no evidence of any mechanisms of death due to cut throat injuries and the only available mechanism of death in this death investigation was emphysema aquosum due to immersion. Therefore, the cause of death was ascertained as drowning. 


\section{Conclusion}

Since there were two suicidal methods, namely cut throat and jumping to river, this should be a complex suicide. Cut throat had been inflicted before entering the water. Theinitial cut throat injuries had failed to cause death and subsequently the deceased succeeded by jumping into river. Therefore, this is a case of an unplanned complex suicide.

\section{References}

1. Demirci S, Dogan KH, Erkol Z, Deniz I. A series of complex suicide. Am J Forensic Med Pathol 2009; 30(2):152-4.

\section{Bohnert M, Complex Suicides. Forensic} Pathology Reviews. Edited by Tsokos M. Vol 2. Totowa, NJ: Humana press; 2005. 127- 43.

3. Kucerova S, Hejna P, Zatopkova L, Straka L. Primary and secondary complex suicide. A 30year retrospective suicide. SoudLek 2012 Jul;57(3):51-5.
4. Saukko P, Knight B. Post-mortem damage by the predators. B. Knight's Forensic Pathology, $3^{\text {rd }}$ ed. Boca Raton USA: CRC press; 2004. 73-6.

5. Vidanapathirana M, Samaraweera JC. Homicidal Cut Throat: The Forensic Perspective. J ClinDiagn Res. 2016; 10(3): GD01-2.

6. Ohshima T, Kondo T. Eight cases of suicide by self-cutting or stabbing: consideration from medico-legal viewpoints of differentiation between suicide and homicide. J Clin Forensic Med.1997; 4 (3):127-32.

7. Solarino B, Buschmann CT, Tsokos M. Suicidal cut-throat and stab fatalities: three case reports. Romanian Journal of Legal Medicine 2011; 19(3): 161-6.

8. Spitz WU. Sharp force injury. In: Spitz WU, Spitz and Fisher's medicolegal investigation of death, 4th ed. Springfield: Charles C Thomas; 2006. pp. 532- 606. 